\title{
Radar probing of surfactant films on the water surface using dual co- polarized SAR
}

\author{
S. Ermakov ${ }^{(1)}$, J.C.B. da Silva ${ }^{(2)}$, I. Kapustin $^{(1)}$, A. Molkov ${ }^{(1)}$, I. Sergievskaya ${ }^{(1)}$, O.Shomina ${ }^{(1)}$ \\ (1) Institute of Applied Physics, Russian Academy of Sciences, 46 Uljanova St., 603950 Nizhny Novgorod, Russia, \\ Email: stas.ermakov@hydro.appl.sci-nnov.ru \\ (2) Department of Geoscience, Environment \& Spatial Planning, University of Porto, Rua do Campo Alegre, 687; \\ 4169-007 Porto, Portugal, E-mail: jdasilva@fc.up.pt
}

\begin{abstract}
Microwave radar is a very perspective tool for all-weather monitoring of film slicks which appear in radar imagery of the water surface as areas of reduced backscattering due to damping of short wind waves. Information about the backscatter variations obtained from single band/one polarization radar seems to be insufficient for film characterization, so, new capabilities of multi-polarization radar for monitoring of film slicks have been actively discussed in the literature. In this paper results of new field experiments on remote sensing of film slicks using dual co-polarized radars: a satellite X-band TerraSAR-X and recently designed at IAP RAS a Multifrequency Radar Complex - three-band scatterometer operating in X-/C-/S-bands and mounted onboard a ship are presented. Along with backscattering depression the variations of polarized (Bragg) and non polarized radar backscatter components in slicks were analyzed. It is obtained that VV-to-HH backscatter ratio is smaller than the ratio predicted by a Bragg (two-scale) model thus indicating that additional, non polarized (NP), component also contributes to the total radar backscatter. Assuming the radar backscatter to be a sum of polarized (Bragg) and NP components the latter was eliminated from the total radar backscatter, and contrasts for the Bragg and NP components were obtained. The contrasts for the polarized component allowed us to estimate damping of gravity-capillary wind waves at Bragg wavelengths in slick and to give more accurate comparison with models of wave damping due to elastic film.
\end{abstract}

Keywords: sea surface, marine slicks, microwave backscattering, dual co-polarized microwave radar, surfactant films, gravity-capillary waves

\section{INTRODUCTION}

Remote sensing of marine film slicks, both biogenic and oil spills, aimed to identify the films and to quantify their characteristics is a very important and urgent problem which, however, is still far from solution. Perspectives of microwave radar as a tool for remote sensing of marine slicks have been actively discussed in the literature (see, e.g. [13] and references therein). Satellite synthetic aperture radar (SAR) is of particular interest for the problem of slick remote sensing because of high SAR resolution. Recently it has been demonstrated in our experiments [4] using one-polarized TerraSAR-X and Envisat ASAR images that depression of radar backscattering (radar contrast) in the range of incidence angles of 20-40 degrees and low-to-moderate wind was not strong (about 2-3 times) and weakly depended both on incidence angle and film elasticity (see, Fig. 1). A widely used composite radar model, taking into account Bragg (resonance) and specular (Kirchoff) scattering mechanisms underestimated radar contrasts at incidence angles less than 25-30 degrees and overestimated the contrasts at larger angles. One of the reasons of the model drawbacks is the existence of an additional, non polarized component of radar return [5], which is associated with wind wave breaking and which seems to appear even at moderate and large incidence angles [6].

Recently attempts to extend capabilities of film slick remote sensing using double- and quad-polarization single band radars has been made in the literature (see, e.g. [7-9]). In [9], in particular it was suggested to analyze different

Remote Sensing of the Ocean, Sea Ice, Coastal Waters, and Large Water Regions 2016, edited by Charles R. Bostater, Jr., Xavier Neyt, Caroline Nichol, Oscar Aldred, Proc. of SPIE Vol. 9999 99990A - (c) 2016 SPIE · CCC code: 0277-786X/16/\$18 - doi: 10.1117/12.2241444 
combinations of $\mathrm{VV}$ and $\mathrm{HH}$ signals, including polarization difference (PD) and polarization ratio (PR), thus elimination either non polarized radar backscatter component or polarized component which respond differently on wave damping due to films. At present time, however, the influence of films on different components of radar return is still not well studied and further experiments are needed to develop models of slick remote sensing. One should note also, that in the majority of reported experiments on radar probing of oil slicks the physical characteristics of the deployed films which are responsible for wave damping were not studied in detail, and this led to strong limitations in theoretical interpretation of the results.

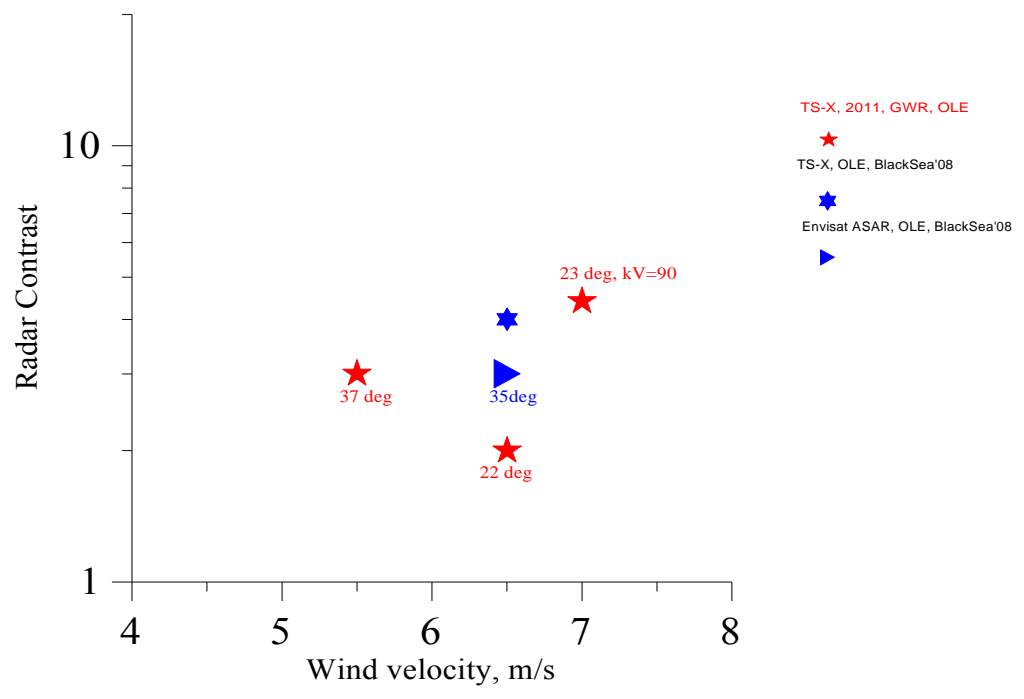

Fig. 1. SAR contrasts (VV-polarization) for OLE slicks at different azimuth and incidence angles (see, [4])

In this paper we present results of new field experiments on radar observations of slicks using dual polarized satellite Terra SAR-X. Additionally, a multiband polarized scatterometer recently made at IAP RAS was used to study characteristics of radar backscattering. Variations of radar return, including its polarized and non polarized components, in slicks with pre-measured physical characteristics of films are analyzed. Comparison of experiment and theory was made.

\section{EXPERIMENT}

Experiments on radar probing of film slicks were carried out in the southern part of the Gorky Water Reservour (GWR), Nizhny Novgorod Region, Russia in 2014-2016. During the experiments of 2014 and 2015 we used data from satellite SAR (TerraSAR-X), provided by DLR. The experiment of 2016 was conducted with a 3-band radar mounted onboard a research vessel. The 3- band radar used in the experiments recently designed in IAP RAS was a Doppler radar, operating in X-, C-, and S-bands at frequencies of $10 \mathrm{GHz}, 6 \mathrm{GHz}$ and $3 \mathrm{GHz}$, respectively, and at two copolarizations $-\mathrm{VV}$ and $\mathrm{HH}$. The radar was mounted onboard a research vessel at a height of about $7 \mathrm{~m}$, and looking at an incidence angle of 60 degrees and to the left at about 40 degrees from the ship's heading.

Characteristics of the experiments are summarized in Table 1. Oleic acid (OLE) was used to deploy monomolecular films on the water surface. Small amounts of these surfactants (about half a liter) dissolved in ethanol were poured on water from a small motor boat moving along a spiral trajectory at least 20 minutes before a satellite pass or before transects by the research vessel. The size of the artificial slicks was typically about 200-300 m.

Wind velocity/direction were measured with an acoustic anemometer WindSonic mounted onboard a research vessel at a height of about $6 \mathrm{~m}$. Physical characteristics of films used in the experiments were studied before the field 
work in laboratory using a method of parametric waves [10]. The method is based on measuring the wavelength and the damping coefficient of gravity-capillary standing waves generated at a certain frequency in a small container and on further retrieval of the surface tension coefficient and dynamic film elasticity, the latter is the main parameter determining damping of wind waves. The elasticity of OLE films grows with surfactant concentration and tends to a constant value for saturated monomolecular film. At higher surfactant concentrations the excess of surface active materials is concentrated in microscopic lenses ("oversaturated" film), and the dynamic elasticity and surface tension in this case are practically the same as for saturated films. OLE films in our experiments were "oversaturated" and could be characterized by constant elasticity and surface tension values, which were about $22 \mathrm{mN} / \mathrm{m}$ and $32 \mathrm{mN} / \mathrm{m}$, respectively.

Table 1. Characteristics of experiments on radar probing of artificial film slicks on the Gorky Water Reservoir.

\begin{tabular}{|c|c|c|c|c|}
\hline Date/sensor & Inc. angle & Wind & Azimuth angle $(\mathrm{kV})$ & $\mathrm{k}_{\text {Bragg }}, \mathrm{rad} / \mathrm{cm}$ \\
\hline 31.08.2014, TerraSAR-X & 37 & $7 \mathrm{~m} / \mathrm{s}, \mathrm{NW}$ & $\mathrm{kV} \approx 40 \mathrm{deg}$. & 2.43 \\
\hline $\begin{array}{l}\text { 03.08.2015, } \\
\text { TerraSAR-X }\end{array}$ & 32.5 & $5 \mathrm{~m} / \mathrm{s}, \mathrm{W}$ & $\begin{array}{l}(\mathrm{kV}) \approx 180 \text { deg. } \\
\text { (upwind) }\end{array}$ & 2.17 \\
\hline $\begin{array}{l}2207.2016, \\
\text { 3band radar, 2nd pass }\end{array}$ & 60 & $7 \mathrm{~m} / \mathrm{s}, \mathrm{E}$ & $(\mathrm{kV}) \approx 40 \mathrm{deg}$. & $1.1,2.2,3.6$ \\
\hline $\begin{array}{l}22 \text { 07.2016, } \\
\text { 3band radar, 3rd pass }\end{array}$ & 60 & $7 \mathrm{~m} / \mathrm{s}, \mathrm{E}$ & $\begin{array}{l}(\mathrm{kV}) \approx 180 \mathrm{deg} . \\
\text { (upwind) }\end{array}$ & $1.1,2.2,3.6$ \\
\hline
\end{tabular}

\section{RESULTS}

Satellite experiment of 31.08.2014.

Fragments of VV and HH polarization images of an OLE slicks in the experiment of 31.08.2014 (TerraSAR-X ascending pass) are presented in Fig. 2. Profiles of normalized radar cross section (NRCS) averaged across the white boxes are shown in Fig. 3 (upper panel).
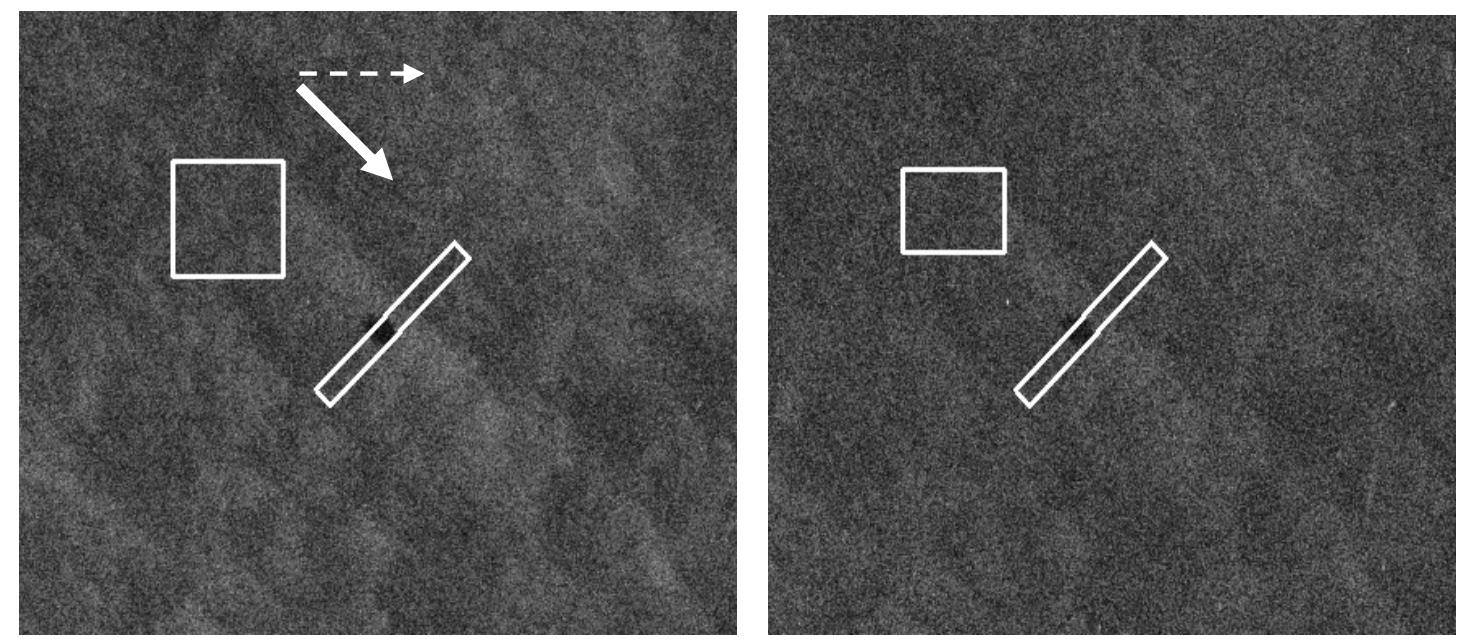

Fig .2. Fragments of VV (left) and HH (right) images of 31.08.2014. Solid and dashed arrows are wind and radar look directions, respectively. 


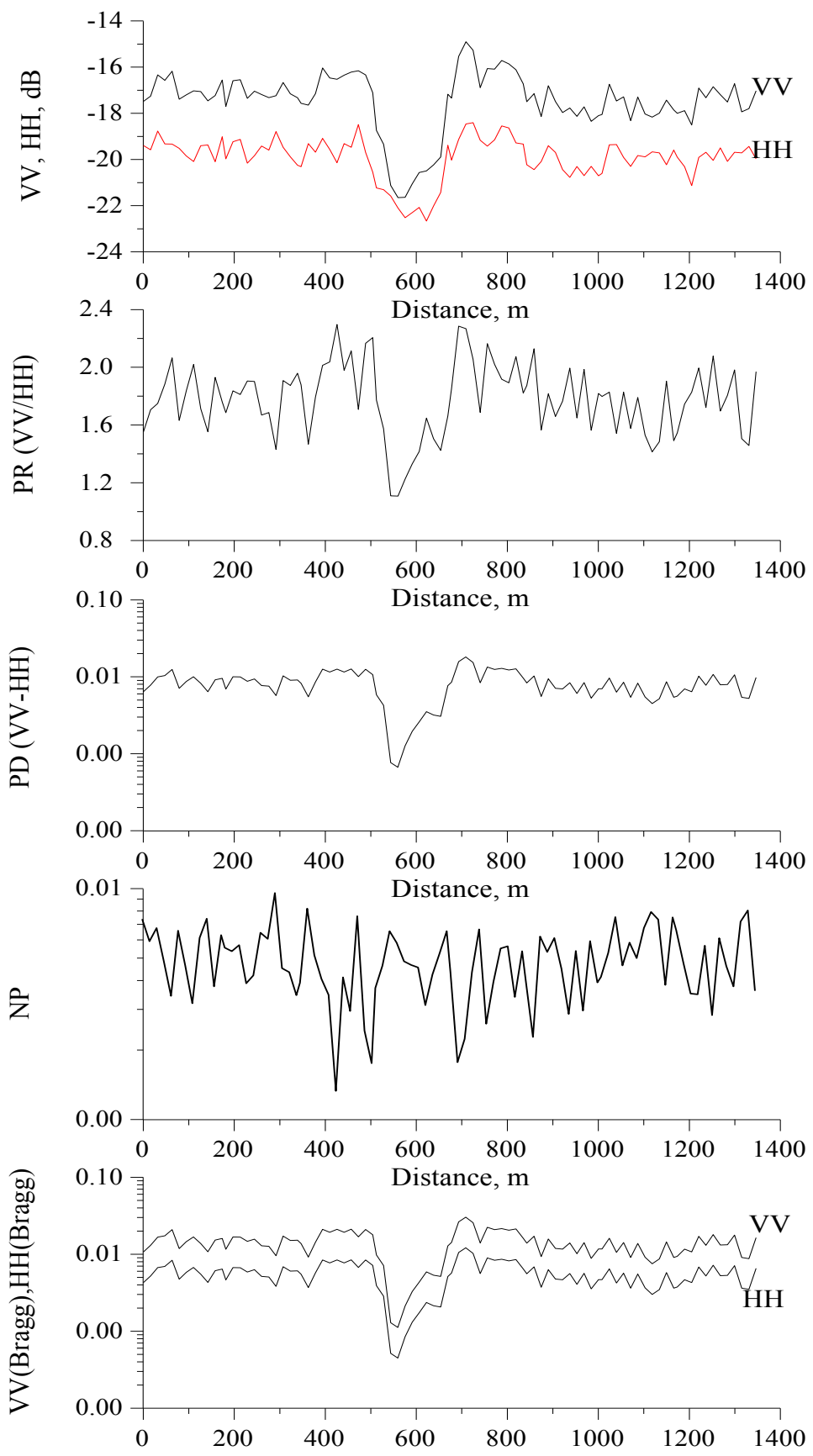

Fig. 3. From top to bottom: TerraSAR-X NRCS at VV and HH polarizations, polarization ratio, relative polarization difference and non polarized and polarized components of radar backscatter along the transects in Fig. 2 


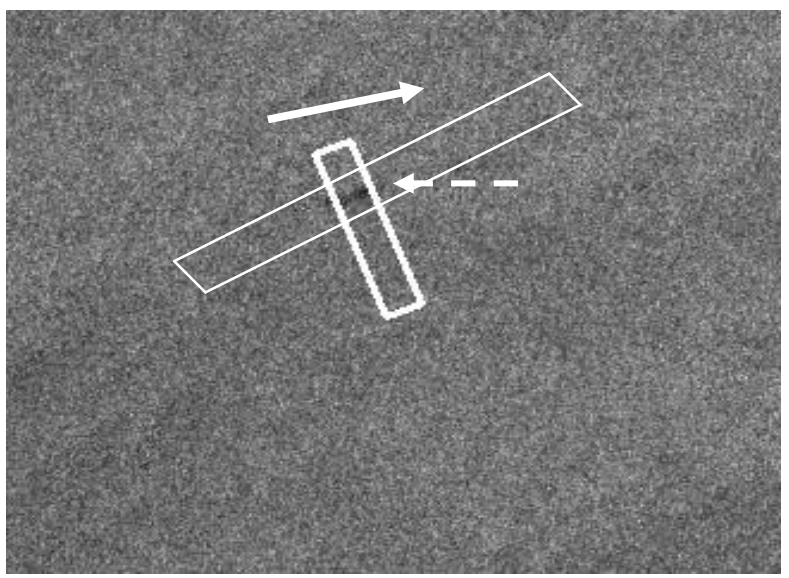

Fig. 4. Fragment of VV polarized image for the experiment of 03.08.2015. Solid and dashed arrows are wind and radar look directions, respectively. Along and cross slick transect boxes are shown schematically.

It is seen from Fig. 3 that NRCS drops in slick for about 4-4.5 dB at VV-polarization and 3-3.5 $\mathrm{dB}$ at $\mathrm{HH}$ polarization. The contrast values are consistent with those obtained in our previous experiments (see, [4] and Fig. 1). Profiles of VV-to-HH radar backscatter ratio ("polarization ratio", PR), VV and HH backscatter difference ("polarization difference", PD), and non polarized (NP) component of NRCS (see, below) are also obtained from NRCS profiles and are shown in Fig. 3.

Satellite experiment of 03.08.2015. A VV-polarized image of OLE-slick is shown in Fig. 4. Two different transects in the image were made: across and along the slick. The radar return characteristics for these transects are quite similar as seen in Fig.5.

Experiment of 22.07.2016 with multifrequency radar. Examples of time series of polarized (VV-HH) and of non polarized radar backscatter components for X-, C- and S- bands for the passes 2 and 3 are presented in Fig. 6. By the time of the pass 3 the slick was partly disrupted due to wind and the first "dip" in the time series corresponds to a small downwind fragment and the second "dip" - to the main slick body. Contrasts for the polarized component are somewhat different for different bands and are quite large, about 10, contrasts for NP component have the same order of magnitude. Contrasts for the pass 2 are about two times smaller, but show similar dependence on Bragg wave number. 

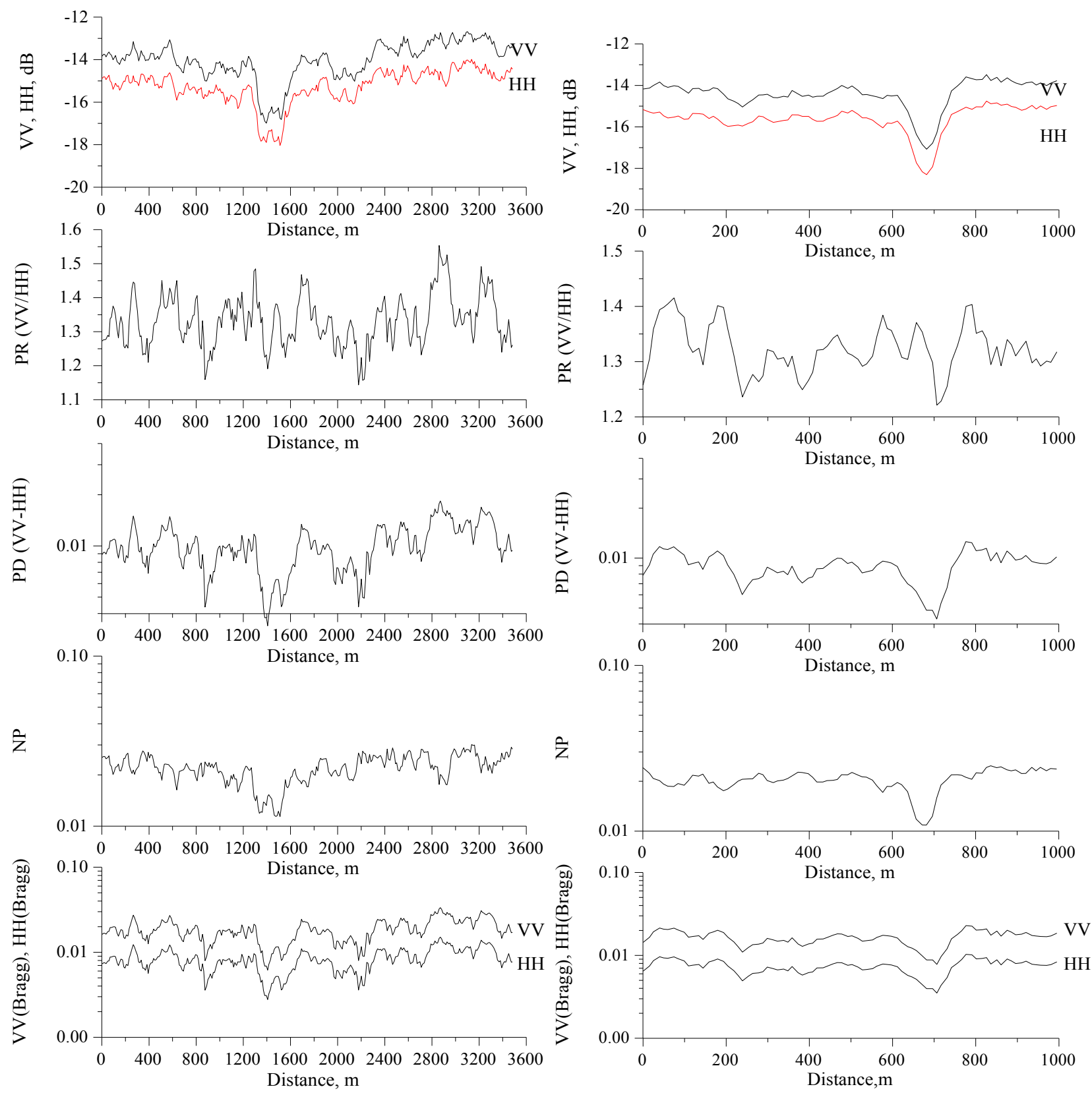

Fig. 5. Same as in Fig. 3 for the along-slick (left) and cross-slick (right) transects. 

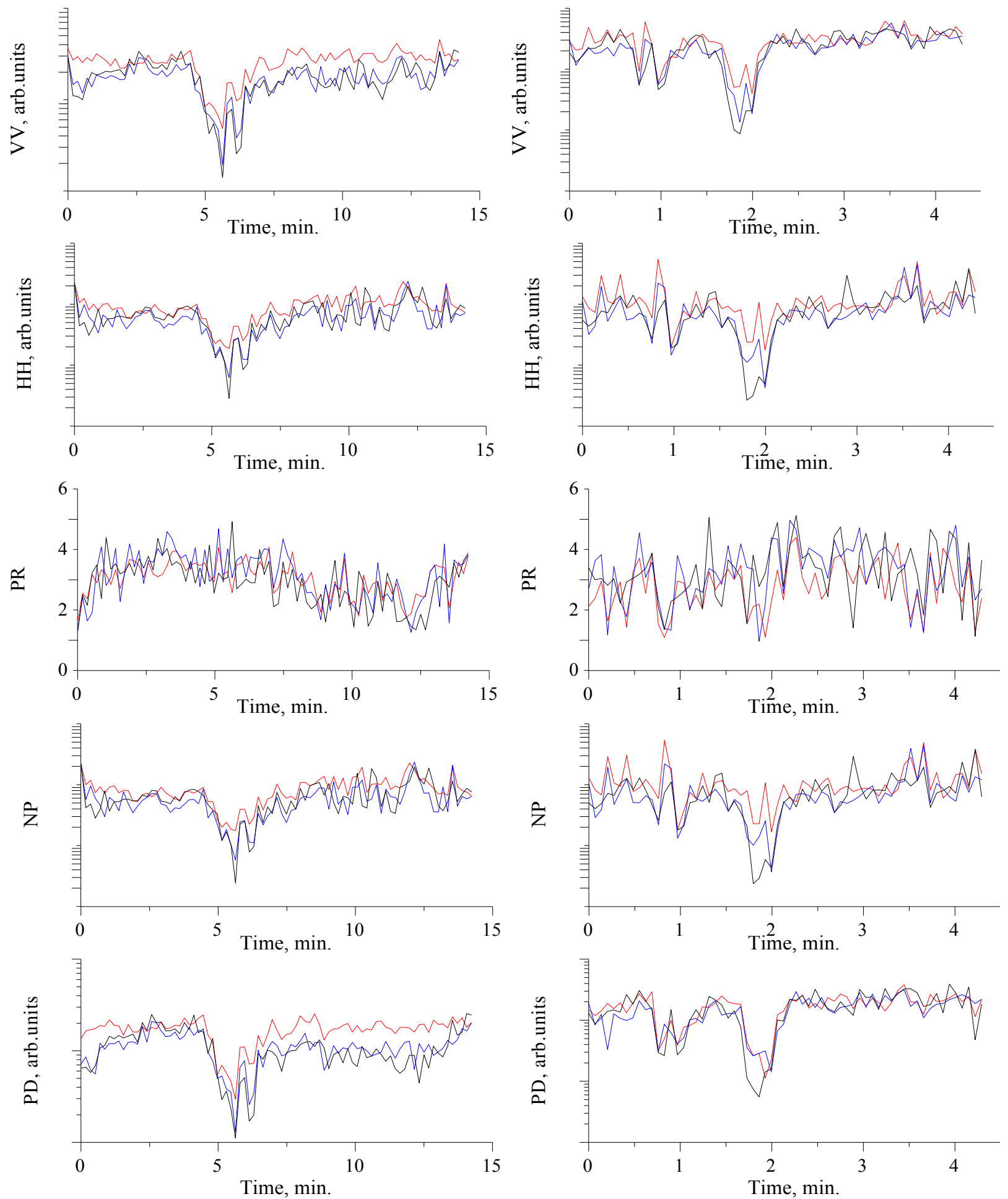

Fig. 6. Same as in Fig. 3 for radar backscatter measured by three band radar. Experiment of 22.07.2016, pass 2- left panel, pass 3 right panel. Red curve corresponds to S-band, blue curve- C-band, black curve - X-band 


\section{DISCUSSION}

It follows from observations that radar backscatter is often roughly described by two-scale Bragg theory, and some additional backscattering mechanism associated with micro- or strong wave breaking can contribute to radar return. So, the total NRCS is supposed to be a sum of Bragg and non polarized components (see, e.g. [5, 9]

$$
\sigma_{p p}^{0}=\sigma_{B_{-} p p}^{0}+\sigma_{N P}^{0}
$$

Where $\sigma_{p p}^{0}$ is the total NRCS, p denotes vertical $(\mathrm{V})$, or horizontal $(\mathrm{H})$ transmit/receive polarizations, $\sigma_{B_{-} p p}^{0}$ is a polarized component of NRCS described by two-scale model, $\sigma_{N P}^{0}$ a non polarized component associated with scattering from small specular elements appeared due to micro or strong wave breaking. The Bragg component, according to two-scale radar model, is proportional to the spectrum of wind waves $F(\vec{k})$ at a Bragg wavevector $\vec{k}_{B}=2 \vec{k}_{\text {radar }} \sin \theta$ ( $\vec{k}_{\text {radaris a }}$ projection of the wavevector of incidence electromagnetic waves on the sea surface, $\theta$ the incidence angle) and the reflection coefficient $R_{p p}(\theta)$ depending on polarization. An attempt to give some empirical description of the NP component, analogous to a Kirchhoff (specular) component, was made in [11].

Assuming (1) one can remove NP component form the total NRCS when deducting $\sigma_{H H}^{0}$ from $\sigma_{V V}^{0}$. Thus obtained polarization difference

$$
P D=\sigma_{B_{-} V V}^{0}-\sigma_{B_{-} H H}^{0}=\left(R_{V V}-R_{H H}\right) F\left(\vec{k}_{B}\right)
$$

is expected to be described by two-scale scattering theory.

Polarization ratio $(\mathrm{PR})$ is defined as

$$
P R=\sigma_{V V}^{0} / \sigma_{H H}^{0}
$$

PR can characterize contribution of NP component to the total NRCS, when compared experimental values of PR with two-scale theory, for which $P R=\mathrm{R}_{\mathrm{VV}} / \mathrm{R}_{\mathrm{HH}}$.

Finally, a non polarized component can be found from (1) and (2) as

$$
N P \equiv \sigma_{N P}^{0}=\frac{1}{2}\left(\sigma_{V V}^{0}+\sigma_{H H}^{0}-\sigma_{B_{-} V V}^{0}-\sigma_{B_{-} H H}^{0}\right)
$$

Radar contrasts in slicks for total NRCS, polarized and non polarized components are defined as

$$
K_{p p}=\frac{\sigma_{p p}^{0}(\text { background })}{\sigma_{p p}^{0}(\text { slick })}, \quad K_{B}=\frac{P D(\text { background })}{P D(\text { slick })}, \quad K_{N P}=\frac{N P(\text { background })}{N P(\text { slick })}
$$

Let us first compare PR values estimated in the described experiment with two-scale theory. Theoretically calculated PR values are shown in Fig. 7. It is clearly seen that experimental PR-values in Fig. 3, 5, 6 are noticeably smaller than theoretical values in Fig.7. This fact indicates that NP component significantly contributes to NRCS. This contribution can be quantitatively estimated from experimental values of NP, VV(Bragg) and HH(Bragg) components presented above. For instance, the HH(Bragg) component in Fig. 3 is nearly the same as NP component, while the VV(Bragg) values are approximately twice as much the NP values. For the experiment of 2015 the background NP component is about twice as the HH (Bragg) component and is almost the same as the VV (Bragg) component. For the 
case of 2016 both NP and VV(Bragg) values are about one order of magnitude the VV (Bragg) values. So, we conclude that contribution of NP component increases with incidence angle compared with HH polarized component.

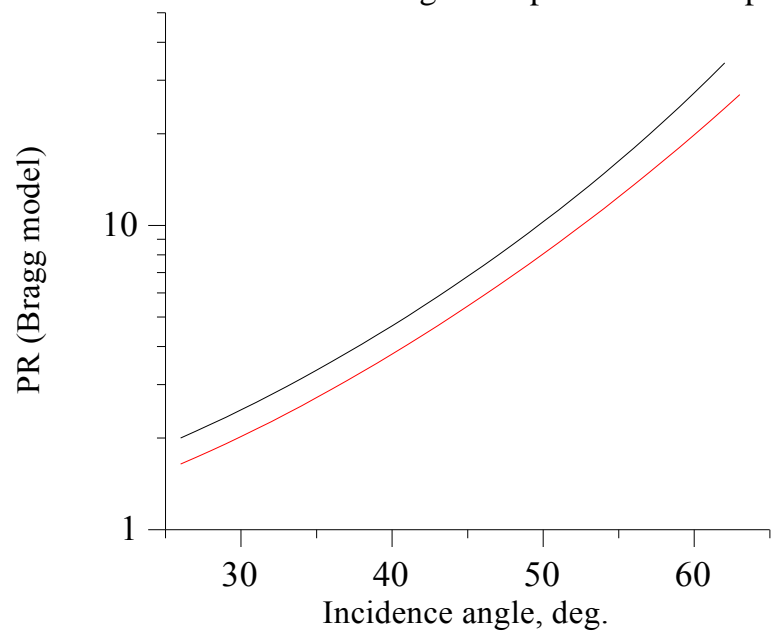

Fig 7. PR dependences calculated according to two-scale Bragg theory (red and black curves are with and without taking into account the influence of long waves, respectively).

The contrast values $K_{V V}$ and $K_{H H}$ in SAR experiments are about 2-3 times, that is consistent with [4], while $K_{B}$-values are about one order of magnitude larger, and the $K_{N P}$ - values are less than 2. At larger incidence angle for the experiment of $2016 K_{B}$ and $K_{N P}$ are quite large, their values are close to each other and depend on radar band.

The obtained $K_{B}$-values for all our experiments are shown in Fig. 8 as functions of Bragg wavenumber. Theoretical dependence of contrast for Bragg waves, calculated according to model [12] for the elasticity of OLE films and wind speed of $7.5 \mathrm{~m} / \mathrm{s}$ is shown in Fig.8, too. One should note that another model (see, [11]) also demonstrates only rough agreement with experiment and further improvement of theory of radar backscatter depression in film slick should be done.

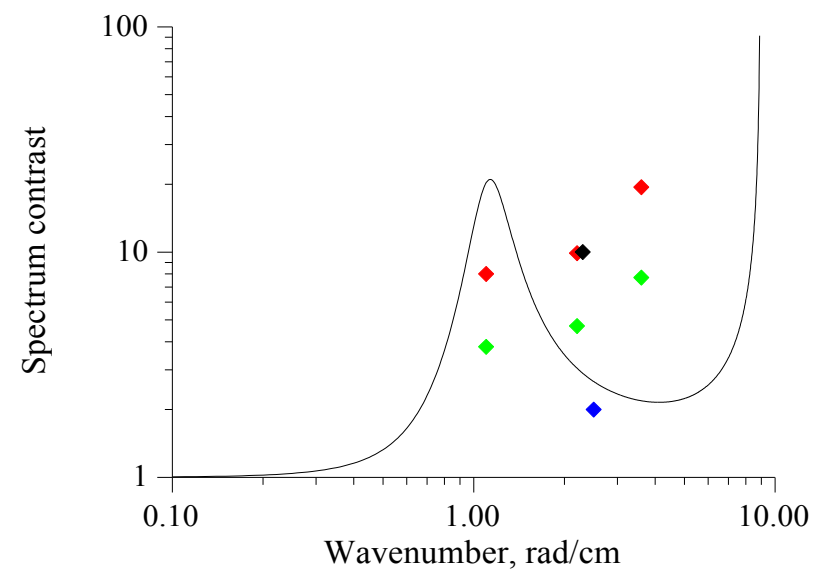

Fig. 8. Contrasts in slicks for polarized NRCS component as function of Bragg wavenumber obtained in TerraSAR-X experiments of 2014 (black dimond) and of 2015 (blue diamond), and in the experiments of 2016 with the three band IAPRAS scatterometer (red symbols - pass 3, green symbols - pass 2). Curve is theory according to model [12] for film elasticity of $20 \mathrm{mN} / \mathrm{m}$ and wind speed of $7.5 \mathrm{~m} / \mathrm{s}$, upwind observation direction. 


\section{ACKNOWLEDGEMENT}

The work was supported by the Russian Ministry of Education and Science (Contract No. 14.607.21.0055, unique identifier RFMEFI60714X0055).

\section{REFERENCES}

[1] Alpers, W., and Huehnerfuss, H. "The damping of ocean waves by surface films: A new look at an old problem," J.Geophys. Res., 94(C5), 6251-6266 (1989).

[2] Scott, J.C., and Thomas, N.H. "Sea surface slicks - surface chemistry and hydrodynamics in radar remote sensing," Wind-overwave couplings. Perspectives and prospects (Sajjadi, Thomas and Hunt, Eds.), Clarendon Press. Oxford, $221-229$ (1999).

[3] Brekke, C., and Solberg, A.H.S. "Oil spill detection by satellite remote sensing", Remote Sensing of Environment, 95, 1-13 (2005) [4] Ermakov S., Da Silva J.C.B., Kapustin I., Sergievskaya, I. "Remote sensing of oil films on the sea surface using radar". Proc. SPIE "Remote Sensing of the Ocean, Sea Ice, Coastal Waters, and Large Water Regions 2012" 85320M. http://proceedings.spiedigitallibrary.org/proceeding.aspx?articleid=1385806 (2012)

[5] Phillips, O. M. "Radar returns from the sea surface-Bragg scattering and breaking waves," J. Phys. Oceanogr., vol. 18, no. 8, 1065-1074 (1988).

[6] Ermakov, S.A., Kapustin, I.A., Kudryavtsev, V.N., Sergievskaya, I.A., Shomina, O.V., Chapron, B., Yurovskiy, Yu.Yu. "On the Doppler Frequency Shifts of Radar Signals Backscattered from the Sea Surface". Radiophysics and Quantum Electronics, Vol. 57, No. 4, 239-250 (2014).

[7] Minchew, B., Jones, C.E., and B. Holt, B. "Polarimetric analysis of backscatter from the Deepwater horizon oil spill using L-band synthetic aperture radar," IEEE Trans. Geosci. Remote Sens., vol. 50, no. 10, 3812-3830 (2012).

[8] Skrunes, S., Brekke, C., and Eltoft, T. "Characterization of marine surface slicks by Radarsat-2 multipolarization features," IEEE Trans. Geosci. RemoteSens., vol. 52, no. 9, 5302-5319 (2014).

[9] Kudryavtsev V., Chapron, B., Myasoedov, A., Collard, F. and Johannessen, J, " On dual co-polarized SAR measurements of the Ocean surface", IEEE Geosci. Remote Sensing Let., 10(4), (2013).

[10] Ermakov S.A., and Kijashko S.V., "Laboratory study of the damping of parametric ripples due to surfactant films", [Marine surface films], Springer, 113-128 (2006).

[11] Kudryavtsev, V., Hauser, D., Caudal, G. and Chapron, B. "A semiempirical model of the normalized radar cross-section of the sea surface: 1. Background model". J.Geophys. Res., V. 108. C3., 8000-8054 (2003)

[12] Ermakov, S.A. [Influence of surfactant films on dynamics of gravity-capillary waves], N. Novgorod, IAP RAS, 1-164 (2010). 